roles) of the Patient-Reported Outcomes Measurement Information System (PROMIS).

Results: Among $100 \mathrm{RD}$ patients, 52 (52\%) had a diagnosis of Rheumatoid Arthritis; 17 (17\%) had a diagnosis of axial spondylorthritis (Ankylosing Spondylitis and Psoriatic Artritis); 25 (25\%) had connectivitis (i.e. Lupus, Systemic Sclerosis, Sjögren Syndrome), and finally 6 (6\%) had vasculitis. Median disease duration was $7 \pm 5$ years. Just under half $(43 \%)$ of RD patients had active disease measured by specific disease activity index. As shown in table 1, no significant difference highlight between the two groups with regard to anthropompetric and demographic characteristics. We found that patients report significantly greater psychosocial changes than healthy controls. More specifically, as shown in figure $1 \mathrm{~A}$, mean $\mathrm{T}$ score for anxiety, depression, fatigue and sleep disturbances were significantly higher in the RD patients than in healthy controls $(56 \pm 9$ vs $48 \pm 8 p<0.001 ; 52 \pm 9$ vs $46 \pm 8 p<0.001 ; 58 \pm 8 v s 48 \pm 8 p<0.001 ; 52 \pm 10$ vs $44 \pm 8 p<0.001)$ respectively. Moreover, also in the social dimension in terms of pain interference, physical functions and satisfaction with participation in social roles, patients showed a median T score worse than healthy controls (Fig.1B).

Table 1

\begin{tabular}{lccc}
\hline & Patients (N=100) & Healthy $(\mathrm{N}=100)$ & $p$ \\
\hline Demographic and & Age (years) & $52.5 \pm 11$ & $51 \pm 18$ \\
Anthropometric & & & \\
ns & Gender n male (\%) & $43(43)$ & $47(47)$ \\
ns & BMl (Kg/m2) & $25.1 \pm 4$ & $27.8 \pm 4$ \\
ns & Smoke $\mathrm{n}(\%)$ & $52(52)$ & $46(46)$ \\
ns & $42(42)$ & $41(41)$ \\
ns & Marital Status n not married (\%) & $31(31)$ & $35(35)$ \\
ns & Occupation n yes (\%) & $54(54)$ & $64(64)$ \\
ns & Education level n degree (\%) & $52(52)$ & - \\
& Rheumatoid Arthritis & $17(17)$ & - \\
& Axial Spondylorthritis & $25(25)$ & - \\
& Connectivitis & $6(6)$ & - \\
& Vasculitis & $7.1 \pm 5.18$ & - \\
& Disease duration (years) & $43(43)$ & - \\
& Disease Activity n yes (\%) & $7(7)$ & - \\
& Medications n (\% patients) & $26(26)$ & - \\
& NSAlD & $54(54)$ & - \\
& Steroids & $34(34)$ & - \\
\hline
\end{tabular}

Continuous variables are shown as mean \pm standard deviation. Categorical variables are presented as number and proportion. The overall $p$-value was calculated by the Mann-Whitney non-parametric test for independent samples and by Chi-square test as appropriate

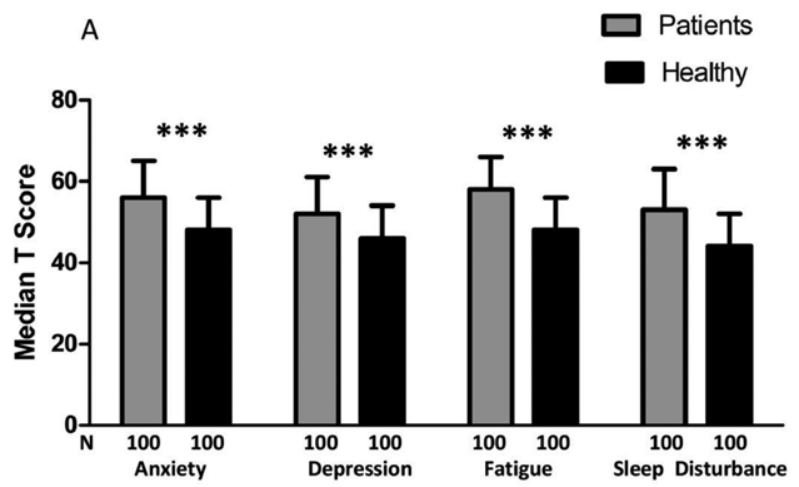

B

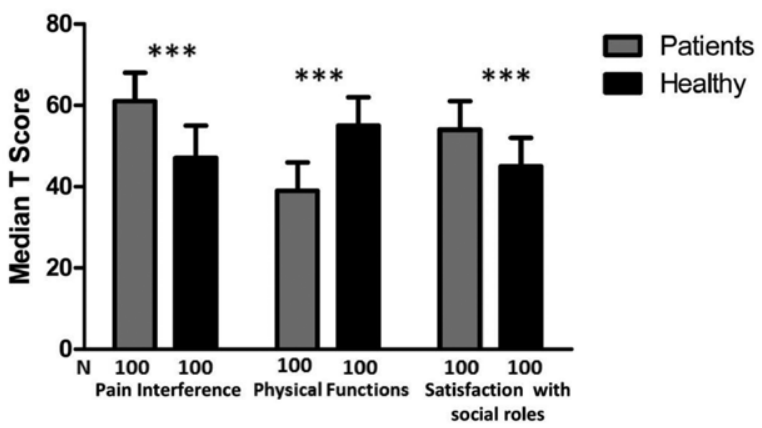

Figure 1. Median T Score stratified by study group. Data are shown as mean and standard deviation. The overall $p$-value wascalculated by the Mann-Whitney non-parametric test for independent samples.
Conclusion: This exploratory study highlights the need to adopt validated questionnaires in clinical practice, and demonstrates that PROMIS is a valid, objective, and standardized instrument that can help nursing staff to better define the consequences of the disease in a patient's daily life.

References:

[1] Minnock P, McKee G, Kelly A, et al. Nursing sensitive outcomes in patients with rheumatoid arthritis: a systematic literature review.Int. J. Nurs. Stud., 77 (2017), pp. 115-129

[2] Bartlett SJ, Orbai AM, Duncan T, et al. Reliability and validity of selected PROMIS measures in people with rheumatoid arthritis. PloS One. 2015

Disclosure of Interests: None declared

DOI: 10.1136/annrheumdis-2020-eular.4493

\section{THU0619-HPR PREVALENCE OF DISTAL INTERPHALANGEAL JOINT ULTRASONOGRAPHY FEATURES IN PSORIATIC ARTHRITIS, SKIN PSORIASIS, OSTEOARTHRITIS AND HEALTHY INDIVIDUALS: A CROSS-SECTIONAL STUDY}

J. Guldberg-Møller ${ }^{1,2}$, M. Henriksen ${ }^{1}$, M. Boesen ${ }^{3}$, L. Dreyer ${ }^{4}$, K. Ellegaard ${ }^{1}$ M. Skougaard ${ }^{1}$, C. Ballegaard ${ }^{1}$, A. L. Tan ${ }^{5}$, R. Wakefield ${ }^{5}$, L. E. Kristensen ${ }^{1}$. ${ }^{1}$ Bispebjerg and Frederiksberg Hospital, The Parker Institute, Frederiksberg, Denmark; ${ }^{2}$ Slagelse Sygehus, Slagelse, Denmark; ${ }^{3}$ Bispebjerg and Frederiksberg Hospital, Department of Radiology, Copenhagen, Denmark; ${ }^{4}$ Aalborg University Hospital, Department of Rheumatology, Aalborg, Denmark; ${ }^{5}$ Leeds Institute of Rheumatic and Musculoskeletal Medicine, Leeds, United Kingdom

Background: Distal interphalangeal (DIP) joint involvement is a feature of both psoriatic arthritis (PsA) and hand osteoarthritis (OA), and nail-changes are features seen both in PsA and nail psoriasis patients without joint involvement (PsO). In both PsA and OA, ultrasonography (US) is used to quantify DIP joint inflammation. Objectives: To explore disease-specific US-detected characteristics in the DIPjoints and extensor tendon entheses in patients with DIP-joint OA, PsA, PsO with nail involvement, and healthy controls $(\mathrm{HC})$.

Methods: In PsA, PsO, OA and HC US examination of DIP joints 2-5 and the extensor tendon were performed. The US images were scored for DIP joint grey-scale synovitis, DIP joint Doppler, osteophytes and erosions (grade 0-3) and presence/absence of enthesitis and peritendonitis of the extensor tendon according to OMERACT standards. Prevalences were calculated on all included fingers (i.e. four fingers per participant), and differences in prevalences were tested using Chi-square statistics.

Results: Fifty PsA patients (44\% females; mean age: $55 \mathrm{y}$ ), $13 \mathrm{PsO}$ patients (38\% females; mean age $54 \mathrm{y}$ ), 12 OA patients ( $100 \%$ females, mean age $71 \mathrm{y})$, and $29 \mathrm{HC}$ (52\% females, mean age 48y) participated. The prevalences across the diagnosis groups are shown in figure 1, and the distribution of US outcomes was significantly different (highest Chi-square P-value: 0.0127 ). The PsA group had the largest prevalence of extensor tendon enthesitis $(45.5 \%)$, peritendonitis (15\%), and DIP joint erosions (11\%), but also exhibited a considerable prevalence of osteophytes (46\%). In the PsO group, the most marked findings were synovitis (33\%) and enthesitis (35\%). The OA group had the largest prevalence of DIP joint synovitis $(67 \%)$ and osteophytes $(88 \%)$ but also $25 \%$ prevalence of enthesitis. $24 \%$ of the $\mathrm{HC}$ group had a grade 1 synovitis.

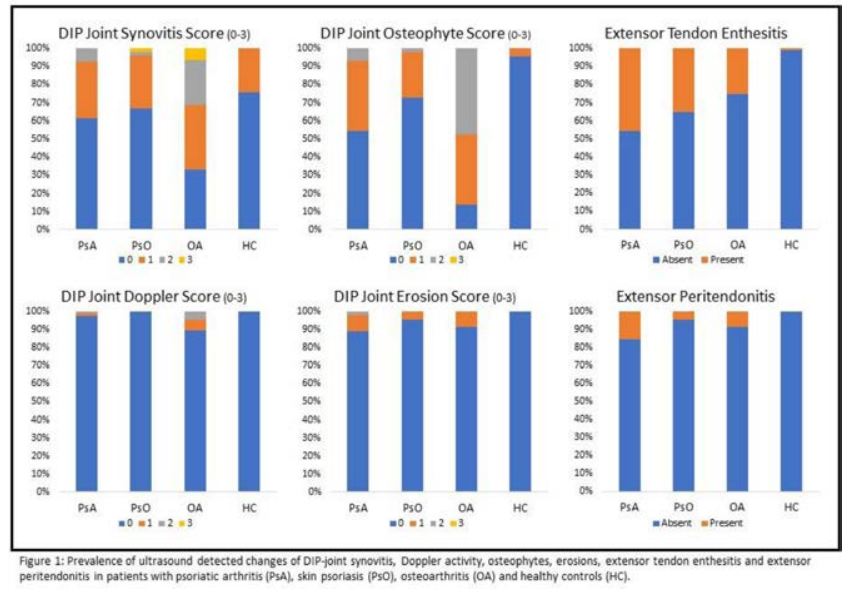

Conclusion: This cross-sectional study found significant patterns of US findings distributed dependent on the underlying condition. PsA patients were mainly differentiated by the presence of extensor tendon enthesitis and peritendonitis. A high prevalence of enthesitis and synovitis was seen in patients 
with DIP joint OA. The high prevalence of enthesitis in $\mathrm{PsO}$ is consistent with a preclinical phase of PsA in this group.

Disclosure of Interests: Jørgen Guldberg-Møller Speakers bureau: Novartis, Ely Lilly, AbbVie, BK Ultrasound, Marius Henriksen: None declared, Mikael Boesen Speakers bureau: Image Analysis Group, AbbVie, AstraZeneca, Eli Lilly, Esaote, Glenmark, Novartis, Pfizer, UCB, Lene Dreyer: None declared, Karen Ellegaard: None declared, Marie Skougaard: None declared, Christine Ballegaard: None declared, Ai Lyn Tan: None declared, Richard Wakefield Speakers bureau: Novartis, Janssen, GE, Lars Erik Kristensen Consultant of: UCB Pharma (Advisory Board), Sannofi (Advisory Board), Abbvie (Advisory Board), Biogen (Advisory Board), Speakers bureau: AbbVie, Amgen, Biogen, Bristol-Myers Squibb,Celgene, Eli Lilly, Gilead, Forward Pharma, Janssen Pharmaceuticals, MSD, Novartis, Pfizer, and UCB Pharma

DOI: 10.1136/annrheumdis-2020-eular.979

\section{THU0620-HPR MEASUREMENT OF LOW DISEASE ACTIVITY USING THE CLINICAL DISEASE ACTIVITY INDEX (CDAI) VERSUS THE DISEASE ACTIVITY SCORE 28 (DAS 28): IMPACT OF INFLAMMATION MARKERS ON THE COMPARATIVE EFFECTIVENESS OF BIOLOGICS FOR THE TREATMENT OF RHEUMATOID ARTHRITIS}

K. Janke ${ }^{1}$, K. Biester ${ }^{1}$, D. Krause ${ }^{2,3}$, B. Richter ${ }^{4}$, C. Schürmann ${ }^{1}$, K. Hirsch ${ }^{1}$, B. Wieseler'. ${ }^{1} / Q W i G$, Cologne, Germany; ${ }^{2}$ Rheumatology Practice Gladbeck, Gladbeck, Germany; ${ }^{3}$ Department of Medical Informatics, Biometry and Epidemiology, University of Bochum, Bochum, Germany; ${ }^{4}$ Cochrane Metabolic and Endocrine Disorders Group, Institute of General Practice, Medical Faculty of the Heinrich-Heine-University Düsseldorf, Düsseldorf, Germany

Background: Biologics for the treatment of rheumatoid arthritis (RA) have different modes of action to target auto-inflammatory processes causing the signs and symptoms of the disease. Different biologics may thus have different effects on inflammatory markers. For instance, previous studies have shown that the interleukin-6-inhibitor tocilizumab (TOC) decreases the level of acute phase reactants (APRs) [1]. Such direct effects on inflammatory markers may lead to an overestimation of clinical response if disease activity is measured via scores including inflammatory markers, such as the Disease Activity Score 28 (DAS 28). The detected changes in disease activity may not adequately reflect the clinical improvement of signs and symptoms.

Objectives: In our study, we compared biologics with each other using two different disease activity scores: the DAS 28 including APRs and the clinical disease activity index (CDAI) excluding APRs. The aim of this study was to assess whether the use of the two different scores affects comparative effectiveness studies on biologics for the treatment of RA.

Methods: We compared results on the comparative effectiveness of biologics using the corresponding thresholds for low disease activity (LDA) for the DAS 28 $(<3.2)$ and the CDAI $(\leq 10)$. We performed two separate network meta-analyses (NMAs) after a thorough step-by-step evaluation of the similarity, homogeneity and consistency assumptions of the patient populations and the study data. Our study formed part of a systematic review (including NMAs) that was largely based on clinical study reports and re-analyses of LDA using individual patient data provided by sponsors for studies conducted up to 2017. Thus, the analyses include hitherto unknown data on LDA analysed by means of the CDAl, especially data from older studies. An extensive comparison of DAS 28 and CDAI in different patient populations was possible.

Results: For all analysed patient populations, comparisons of TOC versus other biologics yielded remarkable results: advantages for TOC were found in NMAs using the DAS 28, which were not confirmed in NMAs using the CDAI. For methotrexate (MTX)-naïve patients, using the DAS 28, TOC showed a greater benefit than abatacept (ABA), certolizumab pegol (CZP), and etanercept (ETA), which was not confirmed by the CDAl. In contrast, TOC showed less benefit than adalimumab (ADA) and ETA. For patients after MTX failure and using the DAS 28, TOC showed a greater benefit than ABA, ADA, anakinra (ANA), ETA, golimumab (GOL), and infliximab (INF). With the exception of ANA, these advantages were not confirmed by the CDAI. Similar differences between DAS 28 and CDAl were shown in patients treated with biologics in monotherapy or after failure of biologics.

Conclusion: In comparative effectiveness studies of biologics, the assessment of LDA using the DAS 28 instead of the CDAl leads to a consistent overestimation of the benefit of TOC in all patient populations, regardless of pre-treatment or combined therapy with MTX. The inclusion of APRs in disease activity scores may thus introduce bias. A score excluding inflammatory markers should therefore be used to ensure valid results.

References:

[1] Smolen JS, Aletaha D. Interleukin-6 receptor inhibition with tocilizumab and attainment of disease remission in rheumatoid arthritis: the role of acutephase reactants. Arthritis Rheum 2011; 63(1): 43-52.
Disclosure of Interests: Kirsten Janke: None declared, Katharina Biester: None declared, Dietmar Krause Grant/research support from: Pfizer and AbbVie (Abbott), Bernd Richter: None declared, Christoph Schürmann: None declared, Katharina Hirsch: None declared, Beate Wieseler: None declared DOI: 10.1136/annrheumdis-2020-eular.4385

\section{THU0621-HPR SARC-F SCALE ON SCREENING SARCOPENIA AND PHYSICAL FUNCTION IN PATIENTS WITH KNEE OSTEOARTRITIS}

M. Karapinar ${ }^{1}$, Z. Baskurt ${ }^{1}$, F. Baskurt ${ }^{1}$, M. Unal ${ }^{2}$, S. Ercan ${ }^{2}$, C. Cetin ${ }^{2}$. ${ }^{1}$ Suleyman Demirel University, Faculty of Health Sciences, Physiotherapy and Rehabilitation Department, Isparta, Turkey; ${ }^{2}$ Suleyman Demirel University, Medicine Faculty, Sports Medicine Department, Isparta, Turkey

Background: Sarcopenia has been defined as a loss of muscle mass and consequently of muscle function. In patients affected by osteoarthritis (OA) a more likely and accelerated development of sarcopenia has been reported. The SARC-F is a simple sarcopenia screening tool includes five assessment items: strength, assistance walking, rising from a chair, climbing stairs, and falls. SARC- $\mathrm{F} \geq 4$ is defined as sarcopenia.

Objectives: The present study aimed to examine the utility of SARC-F in the patients with knee osteoarthritis.

Methods: Patients with radiographic and clinic evidence of tibiofemoral OA (Kellgren-Lawrence score $\geq 2$ ) were included. Sarcopenia were identified using the SARC-F scale. Patients with a total score 4 and higher than 4 were classified as having sarcopenia. Patients were assessed by The Western Ontario and McMaster Universities Osteoarthritis Index (WOMAC), four-meter walking test, hand grip test, shortened version of the falls efficacy scale-international (the short FES-I) and EuroQol- 5 Dimension (EQ-5D). A multiple linear regression model was used to identify independent predictors of SARC-F.

Results: A total 76 patients with median age 61 ranged 55 to 78 years old ( $72.4 \%$ female) were screened in this study (Table 1). Of the sample, $38.1 \%$ of patients were sarcopenic as a SARC-F (Figure 1). Through multiple regression analyses, SARC-F scores was significantly associated with four-meter walking test, hand grip, WOMAC-Function, EQ-5D and short FES-I describing between $57 \%$ and $63 \%$ of the variance (adjusted $\mathrm{R}^{2}$ ) (Table 2). Also, poor physical performance and grip strength were associated with SARC-F $\geq 4$ independently $(\mathrm{P}<0.005)$.

Table 1. Summary Table of Characteristics of sarcopenic vs non-sarcopenic patients with knee OA

\begin{tabular}{lccc}
\hline Measures & $\begin{array}{c}\text { Sarcopenic patients } \\
\text { with knee OA (n:47) } \\
\text { Sarc-F } \geq 4\end{array}$ & $\begin{array}{c}\text { Non-Sarcopenic patients p value } \\
\text { with knee OA (n:29) } \\
\text { Sarc-F<4 }\end{array}$ & \\
\hline Age, mean(SD) & $62.7(6.9)$ & $59.3(6.9)$ & 0.294 \\
BMI, mean(SD) & $27.84(9.56)$ & $28.54(8.38)$ & 0.327 \\
WOMAC, mean(SD) & $43.52(10.83)$ & $28.06(14.9)$ & $0.001^{*}$ \\
GRADE (K-L) med (min-max) & $3(2-4)$ & $2(2-4)$ & $0.008^{*}$ \\
Hand Grip, mean(SD) & $2033(4.89)$ & $28.22(7.13)$ & $0.001^{*}$ \\
4 m Walking Test, mean(SD) & $11.88(4.24)$ & $9.55(4.30)$ & $0.001^{*}$ \\
EQ-5D, mean(SD) & $9.41(1.52)$ & $7.13(1.75)$ & $0.001^{*}$ \\
EQ-5D-VAS, mean(SD) & $44.31(14.12)$ & $66.90(16.25)$ & $0.001^{*}$ \\
Short FES-I, mean(SD) & $18.06(9.45)$ & $9.04(5.32)$ & $0.001^{*}$ \\
Number of falls the past & $4.01(1.24)$ & $3.14(2.74)$ & 0.674 \\
year, mean(SD) & & &
\end{tabular}

$\varphi$ Mann Whitney $\mathrm{U}$ test; ${ }^{*} \mathrm{p}<0.05$; BMI, Body mass index; WOMAC, Western Ontario and McMaster Universities Osteoarthritis Index EQ-5D, EuroQol-5 Dimension; FES-I, Falls Efficacy Scale-International

Table 2. Multiple linear regression analysis for SARC-F

\begin{tabular}{lcccc}
\hline & $\boldsymbol{\beta}$ & $\mathbf{t}$ & P value & $\begin{array}{c}\text { 95\% Confidence Interval } \\
\text { (lower-upper bound) }\end{array}$ \\
\hline Walking Speed & 0.11 & 1.20 & 0.23 & $-0.31 / 0.12$ \\
Hand Grip & -0.35 & -1.73 & 0.04 & $-0.20 / 0.01$ \\
WOMAC-Function & 0.26 & 2.31 & 0.01 & $0.00 / 0.05$ \\
Short FES-I & 0.18 & 1.96 & 0.01 & $0.04 / 0.01$ \\
EQ-5D & 0.22 & 2.29 & 0.02 & $0.02 / 0.39$ \\
EQ-5D VAS & -0.24 & -2.73 & 0.001 & $-0.04 /-0.00$ \\
R $^{2}=0.61$ & & & &
\end{tabular}

WOMAC, Western Ontario and McMaster Universities Osteoarthritis Index, EQ-5D, EuroQol-5 Dimension; FES-I, Falls Efficacy Scale-International 